\title{
Heuristics for Social Games with a Purpose
}

\author{
Aneta Takhtamysheva ${ }^{1}$ and Tim Decker ${ }^{2}$ \\ ${ }^{1}$ Digital Media Lab, TZI, University of Bremen, 28359 Bremen, Germany \\ aneta@tzi.de \\ 2386 Main street, Oneonta, New York 13820, USA \\ timawanderingmanproductions.com
}

\begin{abstract}
Current project introduces a prototype of a game with a purpose constructed using heuristics of social game genre to attract long-term player involvement, and user content generation, to meet the constrains of time and budget.
\end{abstract}

Keywords: social gaming, playful applications, games with purpose, human computation, image tagging.

\section{Introduction}

Social games have experiencing unprecedented success rate. Millions of players, encompassing a large range of age categories play social games to relax, socialize and compete with Facebook friends on daily basis[1]. Social games are known for comparatively small costs of development, minimalistic graphics, simple game mechanics, and viral promotion, that seem to have fit the goals and development facilities of developers of games with a purpose (GWAP) [2].

In the current work we will discuss the heuristics of social games that can be utilized by games with purpose (human computation games) and propose a prototype of social game with purpose that also incorporates mechanisms that allow to deal with the financial and time constraints of researchers.

\section{Social Game Heuristics Principles for Games with Purpose}

Most games with purpose (GWAP) published on social networks so far have not been developed using heuristics specific for social network games; rather they were developed as simple casual games (i.e. Digitalkoot [3]), or gamified playful environments like Sentiment Quiz [4] that utilize social platforms only for the purpose of game deployment. This issue resulted in the lost opportunities of maximizing the reach and engagement of players that social networks like Facebook can potentially offer.

While existing game heuristics frameworks offered by Desurvire[5] and Pinelle [6], cover many elements that are also related to social games, some elements that are related specifically to the social game genre must be added. Paavilainen [7] suggests a 
set of Heuristics for Social Games, based on the guidelines developed by Järvinen [8], which besides the points common to all classic games, includes the following additional elements: rewards for coming back; interruptability; using themes from popular culture; virality and sharing (rewarding players for inviting and gifting friends); narrativity (broadcasting of ingame events as a narration on the player's social network news timeline). Also, other authors mention sophisticated return mechanisms [9] rhythm design [10] ampleness of positive feedback and very clear game visuals and next step guidelines [11] as part of a successful social game model.

We believe that in order to benefit from social game genre, social GWAP should strive to incorporate above mentioned elements whenever relevant.

In addition, Kirman [12] suggest utilizing user empowerment mechanism for asset creation and management of the game, to save efforts, time and money for researchers, and yet creating an engaging experience for players. This tactic has been successfully used in some commercial multi-player online games like Second Life, or Spore and might prove to be a valuable approach in research environment where developers might be limited in expertise, time and budget.

\section{World Architect Game Prototype}

While so far games with purpose were constructed so that the major challenge of the game is the serious tasks itself, we propose a concept of a serious game constructed using the heuristics of social games, where serious tasks are alternated with purely fun tasks.

The goal of this approach is test whether prioritizing fun tasks can leverage the overall sustainability of players' interest to the GWAP and actually increase the number of "serious" contributions of players in the long-run.

We present a concept/prototype of a game called World Architect, which represents a social network game with elements of serious tasks (image tagging). The game consists of variety of quests, some of which are related to serious tasks and others are not but serve the purpose of maintaining the attractiveness of the game.

Players have to set up villages, cities, states and countries by constructing buildings. To avoid costly process of game asset development (buildings) we incorporate user asset generation (Construction Quest). Users should create their own buildings (using graphic tools provided by the game) and can submit those for competitions. Each player is rewarded for participating in the evaluation of submitted pieces (Judgment Quest), the results of which serve as a ground for personalized game reminders. The next Shopping Quest requires users to create shipping tags for construction materials that were purchased to be "transported" to the construction location. The process of creation of shipping tags involves generating image tags. The quality of image tags is verified in the Gardening Quest where players have to match generated tags with images that they were created for. Each successful matching will bring the "gardener" a virtual tree, certain number of which is required to accomplish the construction process, and the "shippers" that created the successful image-tag combination will "safely" receive their construction materials at the construction spot. 
Thus, both sides will reach perfect equilibrium only when providing honest image tagging and validation.

The game environment includes elements that are typical to many existing social network games: a popular culture theme, never-loose multi-session game play; return mechanics; simple strategy genre with simple puzzle elements; user's own unique space with a autonomous management rights; freedom to progress through the game at the desired pace; narrativity; social network login with open access to the user's profile data; and sociability (friends invitations and propagation mechanisms).

In addition to the above, the following guidelines were developed:

- Serious tasks positioned as secondary material to help deliver fun experience to the players rather than being a primary reason for playing the game.

- Serious tasks constructed as light "puzzles" that adds to the engagement of the game.

- Serious tasks deconstructed into smaller pieces and spread among several quests, thus decreasing the risk of breaking the flow of the game [13].

- Game consist of a variety of fairly independent blocks (mini quests) that are connected through storytelling, but can be replaced, improved or removed without destroying the whole game. This makes the game space more flexible for further improvement and extension.

- Mini quests represent a variety of playful activities to chose from - rating, selecting, drawing, puzzle solving, etc, thus giving user freedom of choice in activities instead of limiting to one type of activity.

- The voting scores that each user receives for works submitted for voting competition will in its turn serve two important points: boost efforts and motivation for the players, and:

- Serve as a ground for anticipated updates on how well the submitted works are performing in terms of ratings (narrativity element), and inconspicuously remind players about the game itself.

\section{$4 \quad$ Further Steps}

The next stage of the work is to conduct user studies and test the usefulness of the proposed approach where serious tasks are presented only as secondary elements of social games, and see if proposed guidelines, in particular those related to serious tasks, prove effective in terms of soliciting long term players' contributions. Also, the studies should help to find a proper balance between the number of serious tasks in proportion to the number of playful tasks so that to keep sustainable interest of players while receiving useful input at a pace and quantity that makes the game worth the investments that it required. 


\section{References}

1. PopGames: Social Gaming Research. Research Report. Information Solutions Group (2011), http: / /www. infosolutionsgroup. com

2. Von Ahn, L.: Human computation. In: Proceedings of the 46th Annual Design Automation Conference (DAC 2009), pp. 418-419. ACM, NY (2009)

3. Chrons, O., Sundell, S.: Digitalkoot: Making Old Archives Accessible Using Crowdsourcing. In: HCOMP 2011 Proceedings of the ACM SIGKDD Workshop on Human Computation (2011)

4. Weichselbraun, S., Gindl, A., Scharl, A.: Using games with a purpose and bootstrapping to create domain-specific sentiment lexicons. In: Proceedings of the 20th ACM International Conference on Information and Knowledge Management (CIKM 2011), pp. 1053-1060. ACM, New York (2011)

5. Desurvire, H., Wiberg, C.: Game Usability Heuristics (PLAY) for Evaluating and Designing Better Games: The Next Iteration. In: Ozok, A.A., Zaphiris, P. (eds.) OCSC 2009. LNCS, vol. 5621, pp. 557-566. Springer, Heidelberg (2009)

6. Pinelle, D., Wong, N., Stach, T.: Heuristic evaluation for games: usability principles for video game design. In: Proceedings of the Twenty-Sixth Annual SIGCHI Conference on Human Factors in Computing Systems (CHI 2008), pp. 1453-1462. ACM, New York (2008)

7. Paavilainen, J.: Critical review on video game evaluation heuristics: social games perspective. In: Proceedings of the International Academic Conference on the Future of Game Design and Technology (Futureplay 2010), pp. 56-65. ACM, New York (2010)

8. Järvinen, A.: Game design for social networks: interaction design for playful dispositions. In: Proceedings of the 2009 ACM SIGGRAPH Symposium on Video Games (Sandbox 2009), pp. 95-102. ACM, New York (2009)

9. Thornton, W.M.: 7 - FarmVille, Making Great Games, pp. 101-113. Focal Press, Boston (2011), doi:10.1016/B978-0-240-81285-4.10007-8, ISBN 9780240812854

10. Tyni, H., Sotamaa, O., Toivonen, S.: Howdy pardner!: on free-to-play, sociability and rhythm design in FrontierVille. In: Proceedings of the 15th International Academic MindTrek Conference: Envisioning Future Media Environments (MindTrek 2011), pp. 22-29. ACM, New York (2011)

11. Isbister, K., Schaffer, N.: Chapter 10 - The Strange Case of the Casual Gamer, Game Usability, pp. 143-158. Morgan Kaufmann, Boston (2008)

12. Kirman, B., Casey, S., Lawson, S., Rowland, D.: User Powered Games for Research. In: Proceedings of the Games Design and Technology Workshop and Conference (GDTW), Liverpool (2008)

13. Jones, M.G.: Creating electronic learning environments: Games, flow, and the user interface. In: Proceedings of Selected Research and Development Presentations at the National Convention of the Association for Educational Communications and Technology, AECT (1998) 